\title{
Pathological and DNA-based Detection of Tracheobronchial Tuberculosis in China
}

\author{
Xin $\mathrm{Li}^{1}$, Yi Xie ${ }^{2}$, Yongmei Wang ${ }^{1}$, Junping $\mathrm{Wu}^{3}$, Xin Sun ${ }^{4}$ and Qi $\mathrm{Wu}^{4}$ \\ ${ }^{1}$ Department of Pathology, TCM Key Research Laboratory for Infectious Disease Prevention for State Administration of Traditional \\ Chinese Medicine, Tianjin Haihe Hospital, Tianjin Institute of Respiratory Diseases, Tianjin University, Tianjin, China \\ ${ }^{2}$ Department of Education, TCM Key Research Laboratory for Infectious Disease Prevention for State Administration of Traditional \\ Chinese Medicine, Tianjin Haihe Hospital, Tianjin Institute of Respiratory Diseases, Tianjin University, Tianjin, China \\ ${ }^{3}$ Department of Tuberculosis, TCM Key Research Laboratory for Infectious Disease Prevention for State Administration of Traditional \\ Chinese Medicine, Tianjin Haihe Hospital, Tianjin Institute of Respiratory Diseases, Tianjin University, Tianjin, China \\ ${ }^{4}$ Department of Respiratory Medicine, TCM Key Research Laboratory for Infectious Disease Prevention for State Administration of \\ Traditional Chinese Medicine, Tianjin Haihe Hospital, Tianjin Institute of Respiratory Diseases, Tianjin University, Tianjin, China
}

\begin{abstract}
Objectives: To compare the DNA-based sputum evaluation with histopathology for the diagnosis of tracheo-bronchial tuberculosis (TBTB). Study Design: Case series.

Place and Duration of Study: Tianjin Haihe Hospital, Tianjin Institute of Respiratory Diseases, Tianjin, China, from January to June 2017. Methodology: TBTB patients, who underwent bronchoscopy during the study period, were included. Their bronchoscopy presentations, histopathology, and induced sputum deoxyribonucleic acid (DNA) and culture results were analysed. Results were expressed as frequency percentage.

Results: There were 110 subjects. Most patients were young females with coughing. The main TBTB subtype was fibrostenotic, while the most common findings were single level lesions and lobar bronchi. The diagnostic rates were 40.43\%, 67.86\%, and 68.54\% for DNA-based analysis of induced sputum, histopathology, and induced sputum culture, respectively. Only in the edematous-hyperemic subtype was the positivity rate of DNA-based analysis of induced sputum higher than in histopathology. In the fibrostenotic subtype, the histopathologic results were superior for diagnosis.

Conclusion: A combination of induced sputum and biopsy using DNA-based methods is a superior and exact method to diagnose TBTB. DNA-based analysis of induced sputum for mycobacterium tuberculosis can be used for preliminary impressions.
\end{abstract}

Key Words: Tracheo-bronchial tuberculosis, Mycobacterium tuberculosis, Pathology, Multiplex polymerase chain reaction, Sputum.

How to cite this article: Li X, Xie Y, Wang Y, Wu J, Sun X, Wu Q. Pathological and DNA-based Detection of Tracheobronchial Tuberculosis in China. J Coll Physicians Surg Pak 2021; 31(07):829-832.

\section{INTRODUCTION}

Tracheo-bronchial tuberculosis (TBTB) is a type of tuberculosis in which Mycobacterium tuberculosis (MTB) destroys the mucosa, submucosa, cartilage, smooth muscle, and outer membranes of the trachea and bronchi. ${ }^{1}$ Due to its non-specific manifestations, it may be clinically misdiagnosed as asthma, bronchiectasis, or lung cancer. In terms of histopathology, TBTB can be differentiated from sarcoidosis, Wegener's granulomatosis, or other infectious diseases. ${ }^{2}$

Correspondence to: Qi Wu, Department of Respiratory Medicine, TCM Key Research Laboratory for Infectious Disease Prevention for State Administration of Traditional Chinese Medicine, Tianjin Haihe Hospital, Tianjin Institute of Respiratory Diseases, Tianjin University, Tianjin 300350, China

E-mail: q_hhyy@126.com

Received: May 15, 2020; Revised: March 03, 2021;

Accepted: April 06, 2021

DOI: https://doi.org/10.29271/jcpsp.2021.07.829
TBTB is estimated to occur in $10-50 \%$ of patients with active pulmonary tuberculosis (PTB). Recently, its incidence has been increasing. ${ }^{3}$ TBTB has many complications, such as tracheobronchial stenosis. ${ }^{4}$ Furthermore, its transmissibility is high, underscoring the importance of early diagnosis.

Bronchoscopy is the most useful method in diagnosing TBTB. Presently, bronchoscopic manifestation with histologic features or bacterial findings are considered sufficient to diagnose TBTB. Unfortunately, bronchoscopy is an invasive method that some patients cannot undergo. By contrast, the molecular evaluation of induced sputum (IS) is non-invasive and relatively convenient. However, the molecular testing of IS for TBTB has not been validated, especially in the Chinese population.

In thisstudy, the aim wasto compare DNA-based sputumevaluation with histopathology for the diagnosis of TBTB.

\section{METHODOLOGY}

This was a study of patients with newly diagnosed TBTB in Tianjin Haihe Hospital, Tianjin, China, from January to June, 2017. The inclusion criteria were administration of TBTB in this period. The 
exclusion criteria were patients who could not undergo bronchoscopies. TBTB was defined as the presence of a visible classic endobronchial lesion ${ }^{5}$ (bronchoscopic evidence) as well as meeting one of the following criteria: positive acid-fast stain (AFB) smears from bronchial washing or brushing fluid; pathologic results from the biopsy; positive AFB smear or culture from the sputum; or positive MTB DNA from sputum. TBTB was classified into seven subtypes, based on criteria from Chung et al. At the time of diagnosis, medical histories, high-resolution computed tomography (HRCT) scans, and IS examinations were performed. This study was approved by Tianjin Haihe Hospital Institutional Review Board (No. 201814).

IS was performed by a trained nurse. After inhaling a $5 \%$ hypertonic saline for $15 \mathrm{~min}$, patients were encouraged to cough in order to induce sputum. ${ }^{6}$ Multiplex polymerase chain reaction (MPCR) was used for some patients with suspected TBTB. MPCR was performed by using specific primers to amplify IS6110 segment and MPB64 gene, which respectively had amplicon sizes of 123 and $240 \mathrm{bp}^{7}$

Statistical analyses were performed using Microsoft Excel (version 2010) software. Categorical variables were described as frequencies and percentages, while quantitative variables were expressed as the mean \pm standard deviation (SD).

\section{RESULTS}

The mean age at diagnosis was $41.17 \pm 18.44$ years. The subjects consisted of 75 women and 35 men. The main symptom was cough; few patients were asymptomatic.

Table I: Clinical characteristics of 110 patients with TBTB examined by bronchoscopy.

\begin{tabular}{|l|l|}
\hline Characteristics & $\mathbf{n}(\%)$ \\
\hline Age (years), Mean \pm S.D & $41.17 \pm 18.44$ \\
\hline Incidence by gender & $75(68.18)$ \\
$\quad$ Females & $35(31.82)$ \\
Males & \\
\hline Main symptoms & $95(86.36)$ \\
Cough & $65(59.09)$ \\
Sputum & $41(37.27)$ \\
Fever & $19(17.27)$ \\
Dyspnea & $5(4.55)$ \\
Hemoptysis & $4(3.64)$ \\
Typical tuberculosis toxic symptoms & $4(3.64)$ \\
Asymptomatic & $21(19.09)$ \\
\hline Comorbid disease & $8.17 \pm 15.64$ \\
\hline Symptom duration (Mean \pm S.D, weeks) & $109(99.09)$ \\
\hline Presence of PTB & \\
\hline HRCT findings & $12(10.91)$ \\
Atelectasis & $98(89.09)$ \\
No atelectasis & $25(22.73)$ \\
Cavitary disease & $85(77.27)$ \\
\hline No cavitary disease & \\
\hline
\end{tabular}

Only one patient had no evidence of PTB. According to the HRCT scans, $10.91 \%$ of patients had atelectasis, while $22.73 \%$ had cavities (Tablel).

Most patients had lesions in the lobar bronchi, although 15 had lesions in the trachea, 36 in the main bronchi, and 54 in the segmental bronchi. Most patients had single level TBTB.

The positivity rates of IS culture, biopsy, and IS DNA were,
$68.54 \%, 67.86 \%$, and $40.43 \%$, respectively (Table II). Biopsy positivity rates were relatively high in the actively caseating, fibrostenotic, tumorous, ulcerative, and granular subtypes. Sputum DNA positivity rates were relatively high in the tumorous and granular subtypes. Finally, IS culture positivity rates were relatively high in all subtypes except for granular.

\section{DISCUSSION}

TBTB is a subtype of pulmonary tuberculosis. It is easily misdiagnosed in the clinic due to its poorly understood pathogenesis and non-specific clinical features. Such diagnostic delays increase the possibility of poor outcomes. Most patients with TBTB develop tracheobronchial stenosis, ${ }^{8}$ so it is vital to diagnose TBTB as early as possible to prevent further deterioration and MTB transmission.

As the present data illustrated, the majority of TBTB patients were young women. This gender imbalance may be due to their unique thinner bronchial structures as well as societal customs. Although the average age at TBTB diagnosis in this study was 41.17 years, most patients are diagnosed between the ages of 20 to 40 years. ${ }^{9}$

The main symptom was cough, followed by expectoration, fever, and dyspnea. Diagnosis was often delayed because of the time taken to differentiate this infection from other respiratory diseases. In this study, symptom duration averaged eight weeks. Nearly $100 \%$ of the TBTB patients had active PTB, indicating that active PTB is an important predictor of TBTB occurrence. It remains unclear how to best reduce the onset of TBTB.

In this study, 110 patients underwent HRCT scanning, which detected 12 cases of atelectasis and 25 cases of cavity disease. These findings differed from other reports. ${ }^{10} \mathrm{~A}$ previous study demonstrated that active PTB with cavity lesions was a risk factor for TBTB; cavity lesions are a possible MTB infection pathway. Here, however, cavity disease was not dominant; accordingly, TBTB can occur in the early stages of PTB. Instead, the predominant TBTB subtype in this study was fibrostenotic. Notably, the subtype varies in prior reports, ranging from granular to edematous-hyperemic. ${ }^{9-11}$

Bronchoscopy is a useful, albeit invasive, method to visualise lesions as well as to collect histologic and bacteriological evidence. Biopsy via bronchoscopy is a main method to diagnose TBTB, and the histopathologic results are considered reliable and highly sensitive. Bronchial biopsies are positive in $30-84 \%$ of patients. Even patients with negative sputum smears can be diagnosed with $\mathrm{TB} .{ }^{9}$ Altin et al. reported that, in a group of 50 TBTB patients, 42 cases (84\%) were diagnosed using pathology. ${ }^{12}$ In the present study, the positivity rate was $67.86 \%$, higher than the DNA analyses.

Acommon rapid diagnostic method, used worldwide and recommended by the World Health Organisation, is the microscopic analysis of sputum smears. However, the positivity rate of TBTB from sputum smears is low and variable. 
Table II: TB positive rates of different subtypes of TBTB.

\begin{tabular}{|c|c|c|c|c|}
\hline Subtype & Cases & Biopsy & $\begin{array}{c}\text { Sputum } \\
\text { DNA }\end{array}$ & $\begin{array}{l}\text { Sputum } \\
\text { Culture }\end{array}$ \\
\hline Actively caseating & 27 & $12 / 17(70.59)$ & $2 / 7(28.57)$ & $21 / 25(84)$ \\
\hline Fibrostenotic & 56 & 16/21(76.19) & $11 / 25(44)$ & $27 / 43(62.79)$ \\
\hline Oedematous-hyperaemic & 11 & $1 / 6(16.67)$ & $3 / 8(37.5)$ & $7 / 10(70)$ \\
\hline Tumorous & 8 & $3 / 6(50)$ & $2 / 4(50)$ & $4 / 6(66.67)$ \\
\hline Ulcerative & 3 & $2 / 2(100)$ & $0 / 1(0)$ & $1 / 1(100)$ \\
\hline Granular & 4 & $4 / 4(100)$ & $1 / 2(50)$ & $0 / 3(0)$ \\
\hline Non-specific bronchitic & 1 & $0 / 0(0)$ & $0 / 0(0)$ & $1 / 1(100)$ \\
\hline Overall & 110 & $38 / 56(67.86)$ & $19 / 47(40.43)$ & $61 / 89(68.54)$ \\
\hline
\end{tabular}

Its sensitivity is unsatisfactory due to limitations in specimen-acquisition methods; consequently, the false negative rate is artificially high. Improvements in the accuracy of molecular evaluation methods should be prioritised.

IS is becoming more widely used to obtain sputum specimens. It has been reported to improve the diagnosis of PTB in patients, especially in those with problems producing spontaneous sputum. ${ }^{13,14}$ Nonetheless, the research regarding IS in TBTB remains inadequate. In the data, the DNA IS positivity rate was lower than the biopsy positivity rate. This lower rate may have resulted from a low bacillary load. Moreover, not all patients received MPCR assays. Only in the edematous-hyperemic subtype was the IS DNA positivity rate higher than the biopsy's. This result differed from Hou's report, ${ }^{15}$ perhaps because the histopathologic features of the edematous-hyperemic subtype are not classical. Notably, the rate of histopathologic positivity is highly operator-dependent. By contrast, IS evaluation using DNA-based methods are easier to collect, and the results do not depend on the operator. In the fibrostenotic subtype, the histopathologic results had a higher positivity rate than the IS DNA. However, when bronchi stenosis is present, sputum specimens are difficult to assess.

This study has several limitations. Firstly, the number of patients was small. Secondly, the diagnostic criteria for TBTB was not particularly strict. Thirdly, infection only had to satisfy clinical diagnoses criteria so that the physicians could refer for these investigations. Hence, many cases might have been missed.

\section{CONCLUSION}

Considering all the data, the histopathologic results should be considered sufficient to diagnose TBTB. A combination of IS, biopsy, and bronchoalveolar lavage sample, using DNAbased methods, is the most ideal and accurate approach to diagnose TBTB.

\section{FUNDING:}

This work was supported by the Scientific Fund of Tianjin Haihe Hospital (HHYY-201802).

\section{ETHICAL APPROVAL:}

This study was conducted after the approval of Tianjin Haihe Hospital Institutional Review. Board (No. 201814).

\section{PATIENTS' CONSENT:}

Since it was designed as a retrospective study, the data were collected from the Hospital archive after approval of the Ethics Committee.

\section{CONFLICT OF INTEREST:}

The authors declared no conflict of interest.

\section{AUTHORS' CONTRIBUTION:}

$\mathrm{XL}$ : Concept, design, data analysis, manuscript editing, guarantor.

YX: Conception and design of study, data analysis,

manuscript editing and review.

YW: Conception, data acquisition, manuscript preparation and editing.

JW: Design, data acquisition and analysis, manuscript review.

XS: Design, literature search, manuscript editing.

QW: Conception and design, manuscript editing and review, guarantor.

\section{REFERENCES}

1. Pathak V, Shepherd RW, Shojaee S. Tracheobronchial tuberculosis. J Thorac Dis 2016; 8:3818-25. doi: 10.21037/jtd.2016.12.75.

2. Mondoni M, Repossi A, Carlucci P, Centanni S, Sotgiu G. Bronchoscopic techniques in the management of patients with tuberculosis. Int J Infect Dis 2017; 64:27-37. doi: 10.1016/j.ijid.2017.08.008.

3. Zhang Q, Zhang Q, Sun BQ, Liu C, Su AN, Wang XH, et al. GeneXpert MTB/RIF for rapid diagnosis and rifampin resistance detection of endobronchial tuberculosis. Respirology 2018; 23:950-5. doi: 10.1111/resp.13316.

4. Gil Guerra AB, Gómez San Martín E, López Pedreira MR. Tracheobronchial Tuberculosis. Arch Bronconeumol 2018; 54:41. doi: 10.1016/j.arbres.2017.06.003.

5. Chung HS, Lee JH. Bronchoscopic assessment of the evolution of endobronchial tuberculosis. Chest 2000; 117:385-92. doi: 10.1378/chest.117.2.385.

6. An SQ, Warris A, Turner S. Microbiome characteristics of induced sputum compared to bronchial fluid and upper airway samples. Pediatr Pulmonol 2018; 53:921-8. doi: 10.1002/ppul.24037.

7. Malik S, Sharma K, Vaiphei K, Dhaka N, Berry N, Gupta P, et al. Multiplex Polymerase Chain Reaction for diagnosis of 
gastrointestinal tuberculosis. JGH Open 2018; 3:32-7. doi: 10.1002/jgh3.12100.

8. Faisal M, Harun H, Hassan TM, Ban AY, Chotirmall SH, Abdul Rahaman JA. Treatment of multiple-level tracheobronchial stenosis secondary to endobronchial tuberculosis using bronchoscopic balloon dilatation with topical mitomycin-C. BMC Pulm Med 2016; 16:53. doi: 10.1186/s12890-016- 0209-1.

9. Su Z, Cheng Y, Wu Z, Zhang P, Chen W, Zhou Z, et al. Incidence and Predictors of Tracheobronchial Tuberculosis in Pulmonary Tuberculosis: A Multicentre, Large-Scale and Prospective Study in Southern China. Respiration 2019; 97:153-9. doi: 10.1159/000492335.

10. Guo X, Wang C, Wang X, Ma J, Xv L, Luan T, et al. Characteristics and risk factor analysis of 410 cases of tracheobronchial tuberculosis. Exp Ther Med 2014; 8: 781-4. doi: 10.3892/etm.2014.1804.

11. Siow WT, Lee P. Tracheobronchial tuberculosis: a clinical review. J Thorac Dis 2017; 9:E71-E77. doi: 10.21037/jtd.
2017.01.49.

12. Altin S, Cikrikçioglu S, Morgül M, Koşar F, Ozyurt H. 50 endobronchial tuberculosis cases based on bronchoscopic diagnosis. Respiration 1997; 64:162-4. doi: 10.1159/0001 96662.

13. Atwebembeire J, Orikiriza $P$, Bonnet $M$, Atwine D, Katawera $V$, Nansumba $M$, et al. Xpert( $\left({ }^{B}\right)$ MTB/RIF for detection of Mycobacterium tuberculosis from frozen string and induced sputum sediments. Int J Tuberc Lung Dis 2016; 20:1113-7. doi: 10.5588/ijtld.15.0691.

14. Zar HJ, Workman LJ, Prins M, Bateman LJ, Mbhele SP, Whitman CB, et al. Tuberculosis Diagnosis in Children Using Xpert Ultra on Different Respiratory Specimens. Am J Respir Crit Care Med 2019; 200:1531-8. doi: 10.1164/rccm. 201904-07720C.

15. Hou G, Zhang T, Kang DH. Efficacy of real-time polymerase chain reaction for rapid diagnosis of endobronchial tuberculosis. Int J Infect Dis 2014; 27:13-7. doi: 10.1016/j.ijid.2014.04.027. 